\title{
Incorporation of Additives in Single Crystals - Bio-inspired approach
}

Yi-Yeoun Kim $^{1}{ }_{\llcorner}$Alex Kulak ${ }^{1}$, Pengcheng Yang ${ }^{2}$, Mona Semsarilar ${ }^{2}$, Joseph Carloni ${ }^{3}$, and Shefford Baker $^{3}$, Steven Armes ${ }^{2}$ and Fiona Meldrum ${ }^{1}$

${ }^{1}$ School of Chemistry, University of Leeds, Leeds LS2 9JT, UK

2 Department of chemistry, University of Sheffield, Sheffield, S3 7HF, UK

${ }^{3}$ Department of Materials Science and Engineering, Cornell University, Ithaca, New York 14853, USA

Additives are widely used means of controlling the size, shape and structure of crystalline materials. Formation of biogenic crystals is a beautiful example of how to achieve finely tuned crystal growth occurred in the rich additive environments. A key characteristic of the biominerals is their nano-composite structure, which derives from intimate association of organic molecules with the mineral host.

This talk will discuss how bio-inspired approaches can be used to generate new class of nanocomposite with tunable compositions and properties. Single crystals of calcite as a host mineral were precipitated in the presence of "guest species" ranging from commercial latex particles, to organic/inorganic nanoparticles functionalized with block copolymers,[1-3] to finally small molecules like amino acids[4].

Control over sizes and surface chemistry/structure of guest species and growth solution conditions determined the efficiency of incorporation, leading to tunable occlusion level from 0 to $7 \mathrm{~mol} \%$ for amino acids[4] and 10-20 $\mathrm{wt} \%$ for nanoparticles,[1-3] without disturbing single crystal structure (measured using HPLC, TGA and AA). Further, the internal and external structures of the resultant composite crystals were characterized in combination of Raman/IR spectroscopy, high resolution powder XRD, FIB-sectioned SEM and TEM. Finally the mechanical properties were characterized with nano-indentation. The synthetic crystals containing organic species exhibit analogous texture and defect structures to biogenic calcite crystals and are harder than pure calcite. Extension of these studies to the incorporation of inorganic nanoparticles [3] in other host single crystals including $\mathrm{ZnO}, \mathrm{MnCO}_{3}$ and $\mathrm{Sr} / \mathrm{BaSO}_{4}$ ) again demonstrated high levels of incorporation with additional magnetic or optical properties.

This simple crystal growth methodology provides a unique model for giving insight into both the mechanism of occlusion of within single crystals, and the relationship between the macroscopic properties of a crystal and its microscopic structure.

[1] Kim Y-Y. et al (2011) Nature Mater. "Artificial Biominerals - Incorporation of Copolymer Micelles in Calcite Single Crystals" 10, 890-896.

[2] Kim Y-Y. et al (2016) Adv. Funct. Mater. "Structure and Properties of Nanocomposites Formed by the Occlusion of Block Copolymer Worms and Vesicles within Calcite Crystals"

[3] Kulak A. N. et al (2016) Chem. Mater. "Polymer-Directed Assembly of Single Crystal Zinc Oxide/Magnetite Nanocomposites under Atmospheric and Hydrothermal Conditions"

[4] Kim Y-Y. et al (2016) Nature Mater. "Tuning hardness in calcite by incorporation of amino acids" 